\title{
Encoding and Recognition Processing of Chinese Characters: A Functional Magnetic Resonance Imaging Study
}

\author{
Jinlong Zheng, ${ }^{1}$ Siyun Shu, ${ }^{2}$ Bin Wang, ${ }^{2}$ Xiangyang Tian, ${ }^{1}$ Xinmin Bao, ${ }^{2}$ Yongming Wu, ${ }^{3}$ \\ Zengqiang Zhang, ${ }^{4}$ Xiangyang Cao, ${ }^{1}$ and Lin $\mathrm{Ma}^{5}$ \\ ${ }^{1}$ Department of Neurology, Huaian First People's Hospital, Nanjing Medical University, Huaian, Jiangsu 223300, China \\ ${ }^{2}$ Center of Pediatrics, Zhujiang Hospital, Southern Medical University, Guangzhou, Guangdong 510282, China \\ ${ }^{3}$ Department of Neurology, Nanfang Hospital, Southern Medical University, Guangzhou, Guangdong 510515, China \\ ${ }^{4}$ Institute of Geriatrics and Gerontology, Hainan Branch of Chinese PLA General Hospital, Sanya, Hainan 572014, China \\ ${ }^{5}$ Department of Radiology, PLA General Hospital, Beijing 100853, China
}

Correspondence should be addressed to Siyun Shu; shusiyun@126.com and Lin Ma; cjr.malin@vip.163.com

Received 2 September 2015; Accepted 30 November 2015

Academic Editor: John H. Zhang

Copyright (C) 2016 Jinlong Zheng et al. This is an open access article distributed under the Creative Commons Attribution License, which permits unrestricted use, distribution, and reproduction in any medium, provided the original work is properly cited.

\begin{abstract}
This study aimed to investigate the conceptual memory processes that underlie encoding and recognition processing of Chinese characters. Healthy participants $(n=14)$ performed a semantic-relatedness paradigm using categorically related logogram pairs from four different categories (fruit, animal, tool, and clothing). During intentional encoding, subjects were instructed to make semantic judgments and select category-correlated features to bind and memorize logogram pairs. During recognition, subjects were asked to recognize the memorized items. The MATLAB software and spatial clustering analysis were used for image data processing. Compared with baseline, encoding mainly activated BA13, with significant effects in BA6/8/9/46/45/47, BA24, BA7/39/40, BA37/20, and BA18/19; meanwhile, recognition mainly activated BA6/8/9/10/13/45/46/47, BA31, BA7/40, and BA18/19. Compared with recognition, encoding activated BA18/19/37/20/36 with a peak activation area in BA18. Compared with encoding, recognition significantly activated BA7, BA31/32, and BA10. In conclusion, distributed networks of discrete cortical regions with distinct roles are active during semantic processing of logograms. The ventral occipitotemporal and inferior frontal regions display increased levels of encoding-related activity. The dorsal medial brain regions, including the superior frontal gyrus and occipitoparietal regions, are associated with recognition-related activity.
\end{abstract}

\section{Introduction}

Conceptual memory is involved in the general knowledge of concepts, objects, facts, people, and word meaning. Recent neuroimaging studies in conceptual knowledge begin to reveal that specific functions are performed within small areas of the brain, with these small areas involved in a hub or network that also includes areas of the brain responsible for perceiving and acting [1]. Conceptual knowledge is usually shared by individuals from the same culture, with some variations according to each individual experience.

Alphabetic languages and logographic Chinese are markedly different because they represent the meaning of words in very different ways. Indeed, alphabetic words are read by assembled phonology, while Chinese characters are read as a concept by their visual shapes, without involvement of phonemes [2]. A previous study revealed that phonological processing of Chinese characters occurs in Brodmann area (BA) 9, BA46, and BA40, with minor activation of BA45/47, while phonological processing in native English speakers is made in BA44/45 and BA22 [3].

However, the organization of this knowledge within the brain is controversial. Indeed, there are two theories about this: the distributed-only and distributed-plus-hub views, respectively [1]. Studies revealed that recognizing specific knowledge activates specific areas: colors and forms activate the left ventral temporal cortex, whereas recognition of size information activates the parietal cortex [4-6]. Tasks such as 
animal naming are uniquely associated with the left medial occipital cortex, whereas tool naming specifically involves the left premotor and posterior middle temporal cortices $[7,8]$.

Recent studies have provided strong evidence that the human conceptual system is not organized by category specificity $[9,10]$. It has been postulated that the organization of semantic representations has more than one governing principle. It is important to note that the activity associated with each object-specific category involves a relatively large expanse of brain areas, suggesting that representations of different categories are distributed and overlapping [1]. It is possible that feature-based models provide the flexibility needed to represent an infinite variety of object categories [11, 12].

Few studies have examined the brain areas involved in the processes of encoding and recognizing conceptual information regarding Chinese characters. Therefore, the aim of the present study was to use functional magnetic resonance imaging (fMRI) to study the brain areas involved in encoding and recognizing conceptual information concerning Chinese characters.

\section{Materials and Methods}

2.1. Subjects. Fourteen native Mandarin Chinese speakers, including seven males and seven females, were recruited to participate in the semantic category memory test. All subjects were right-handed as determined by the handedness inventory [13]. Participants were all undergraduate students from the Southern Medical University, Guangzhou, China. Participants ranged in age from 20 to 23 years and had normal or corrected-to-normal vision. This study was approved by the Medical Ethics Committee of Nanfang Hospital. All participants provided written informed consent before enrolment, in accordance with the Declaration of Helsinki. The subjects underwent a Clinical Dementia Rating (CDR) test. Inclusion criteria were (1) no memory disturbances, (2) normal physical status, and (3) $\mathrm{CDR}=0$. Subjects who had a history of neurological or psychiatric illness condition or who were taking any medications immediately prior to or during the scans were excluded from the study.

2.2. Chinese Characters. All test logograms (visually presented in pairs to each subject) were selected from the Modern Chinese Dictionary [14]. All logograms were used commonly and had a frequency of occurrence of no less than 30 per million, according to the Modern Dictionary of Frequently Used Chinese [15]. In each logogram pair, the first one was regarded as the stimulating logogram, while the second was considered the responding logogram. Four groups of semantic categories (fruits, animals, tools, and cloths) were selected for this study, using four stimulating and four responding logograms from the same category, alongside eight other logograms from similar categories. By combinations and repetitions, a total of sixty-four logograms were selected for each category memory task.

2.3. fMRI. The experiment was performed using a 1.5 Tesla Magneto scanner (Siemens, Sonata, Germany) equipped with a fast gradient system for echo-planar imaging (EPI). A standard radio frequency head coil was used. Head motion was restricted, with ear plugs used to reduce scanner noise. Visual stimuli were presented to subjects by projecting the video display of computer onto a translucent screen. Subjects viewed the stimuli through a mirror attached to the head coil. The presentation time of stimuli was accurately controlled by the DMDX display software (http://www.u.arizona.edu/ $\sim \mathrm{kforster} / \mathrm{dmdx} / \mathrm{dmdx}$. htm). Before the tests, subjects were visually familiarized with the procedures and test conditions to minimize anxiety and to enhance task performance. EPI was performed using a gradient-echo with scan parameters of repetition time (TR)/echo time (TE)/flip angle = $2,500 \mathrm{~ms} / 25 \mathrm{~ms} / 90$ degrees. The acquisition of the matrix was $64 \times 64$ in each plane, with a field-of-view (FOV) of $210 \mathrm{~mm}$ $\times 210 \mathrm{~mm}$. Thirty contiguous axial slices (slice thickness $=$ $4 \mathrm{~mm}$ ) parallel to the AC-PC line were acquired to cover the whole brain. Anatomical MRI was acquired using a T1weighted $3 \mathrm{D}$ gradient-echo pulse-sequence, which provided high resolution $\left(1 \times 1 \times 1 \mathrm{~mm}^{3}\right)$ images of the entire brain.

2.4. Presentation of Logogram Pairs. All logograms were presented to the subjects in Chinese using the same font and size. A block design was used. The test in each subject contained four cycles composed of four blocks: 25-second (s) block of a memory-encoding condition, $25 \mathrm{~s}$ block of a recognition condition, 25 s block of a baseline ${ }_{1}$ condition, and $25 \mathrm{~s}$ block of baseline ${ }_{2}$ condition. Each block consisted of an image reminding the patients of the testing process displayed for $1 \mathrm{~s}$, followed by eight logogram pairs displayed for $3 \mathrm{~s}$ each (Figure 1). Each cycle covered one category: fruits, animals, tools, or cloths.

During each memory-encoding condition block, subjects were asked to identify and memorize four logogram pairs that belonged to the same category (e.g., fruit group test: grape-cherry), and these were mixed with other four similar logogram pairs (e.g., tomato-cucumber). During intentional encoding, subjects were instructed to make semantic judgments and select category-correlated features to bind and memorize logogram pairs; that is, subjects were instructed to judge the same group of logogram pairs from the presented eight pairs using their own conceptual knowledge (e.g., size, color, and form) to find the stimulating logogram and responding logogram and memorize them. Each logogram pair was presented randomly for $3 \mathrm{~s}$.

In each recognition condition block, all pairs of logograms shared the same category (e.g., fruit group). There were four logogram pairs presented that were exactly the same as during the encoding condition; the other four logogram pairs were recombined from the same group (e.g., grape-cherry and grape-pear). Examples of stimuli for the fruit group test are presented in Figure 1. The logogram pairs appeared in a random order. Subjects were required to recognize four pairs of logograms just memorized during the encoding condition. Subjects indicated their logogram pair selection by pressing a key button with the index of the right (dominant) hand.

In each baseline ${ }_{1}$ condition block, a fixation logogram pair (e.g., apple-apple) was adopted in order to create a condition in which no memorization or recognition was 


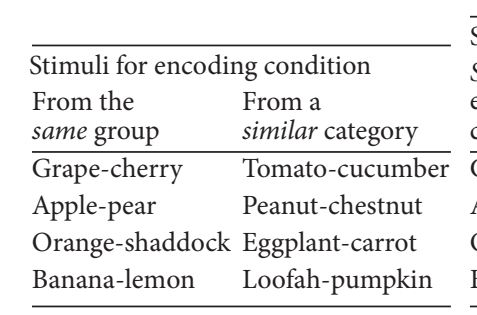

\begin{tabular}{ll}
\hline $\begin{array}{l}\text { Stimuli for encoding condition } \\
\text { Same as }\end{array}$ & $\begin{array}{l}\text { Different from } \\
\text { encoding }\end{array}$ \\
encoding & condition \\
condition & Grape-pear \\
\hline Grape-cherry & Apple-cherry \\
Apple-pear & Orange-shaddock \\
$\begin{array}{ll}\text { Orange-lemon } \\
\text { Banana-lemon }\end{array}$ & Banana-shaddock \\
\hline
\end{tabular}

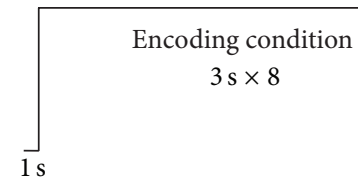

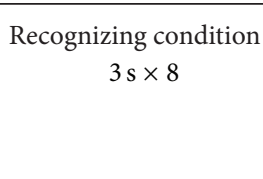

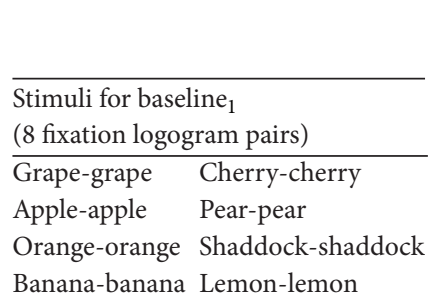

\begin{tabular}{ll}
\hline $\begin{array}{l}\text { Stimuli for baseline } \\
\text { Fixation }\end{array}$ & $\begin{array}{l}\text { Responding } \\
\text { changing }\end{array}$ \\
logogram & logogram pairs \\
pairs & Grape-pear \\
\hline Grape-grape & Apple-cherry \\
Apple-apple & Orange-lemon \\
Orange-orange & Orang-shaddock \\
Banana-banana & Banana-shad
\end{tabular}

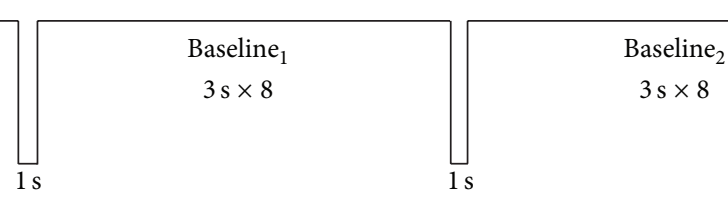

FIGURE 1: Design of one stimulation cycle. Each cycle contained four blocks. Each block was preceded by a $1 \mathrm{~s}$ period during which an image was presented to remind the patient to the study principle. Then, eight pairs of logograms were displayed for $3 \mathrm{~s}$ each. The figure used the fruit category as an example. The logogram pairs appeared in a random order.

needed. The fixation logogram pair was replaced every $3 \mathrm{~s}$, and subjects were instructed to maintain fixation on the logogram pair.

In each baseline ${ }_{2}$ condition block, four fixation logogram pairs (e.g., apple-apple) were presented together with four other logogram pairs, with the responding logogram different from the stimulating one (e.g., apple-cherry). The button had to be pressed when the fixation pair appeared.

2.5. Data Analysis. The MATLAB software (MathWorks, Inc., Natick, MA, USA) and SPM8 (http://www.fil.ion.ucl.ac .uk/spm) were used for image data processing. Image preprocessing was performed using the Data Processing Assistant for Resting-State fMRI (DPARSF) V1.0 software (http://restfmri.net/forum/DPARSF). DPARSF is a plug-in software package based on SPM (http://www.fil.ion.ucl.ac.uk /spm) and RS-fMRI Data Analysis Toolkit (REST, by Song et al., http://www.restfmri.net) [16]. Functional data preprocessing included slice timing correction, motion correction, spatial normalization, and smoothing $(\mathrm{FWHM}=4 \mathrm{~mm}$ ). Data with head motions of more than $2.0 \mathrm{~mm}$ maximum displacement in any of the $x, y$, or $z$ directions or 2.5 degrees in any angular motion were discarded.

Preprocessing of fMRI data was performed with a time-series of images acquired from the same subject using the least squares approach and six-parameter (rigid body) transformation to remove movement artifacts. The images were spatially smoothed with an $8 \mathrm{~mm}$ Gaussian kernel to decrease spatial noise. Skull stripping of the 3D MRI T1-weighted images was carried out with the Alice software (Perceptive Systems, Inc., Boulder, CO, USA). A generalized linear model approach was used in each participant according to image groups (encoding, recognition, baseline ${ }_{1}$, and baseline $_{2}$ ). Activation maps were calculated by comparing images acquired during the task with control conditions (i.e., the encoding condition was compared with baseline $e_{1}$ and the recognition condition with baseline $e_{2}$ ) using Student's $t$-tests. One-sample $t$-tests were performed on activation maps using SPM8 (http://www.fil.ion.ucl.ac.uk/spm). Multiple comparison corrections for the data were performed using the
Monte Carlo simulation (AlphaSim by B. Douglas Ward, http://afni.nimh.nih.gov/pub/dist/doc/manual/AlphaSim.pdf). Combination threshold of voxel was $P<0.05$, and a cluster size $>389$ corresponded to a corrected $P<0.05$.

The activation maps of all 14 subjects were then overlaid on the corresponding T1 images using random effect analysis [17].

MNI coordinates were converted to Talairach coordinates using nonlinear transformation. Coordinates shown in Talairach space for the center-of-mass and volume $\left(\mathrm{mm}^{3}\right)$ of each activation cluster were determined based on the averaged activation maps. Anatomical labels and BAs were identified according to the Talairach Daemon database. The regions that showed significant differences were extracted as regions of interest (ROIs).

\section{Results}

The detailed brain regions of significant activation for encoding versus baseline $e_{1}$ are listed in Table 1 and presented in Figure 2. When comparing encoding with baseline $e_{1}$, large clusters of activation were located in right prefrontal cortex with a peak activation area in insular cortex (BA13) extending to the midinferior frontal region (coordinates: $36,24,7, t$ value $=9.20$, and $P<0.05$ ). Significant activations included three clusters in different regions: (1) right prefrontal cortices (BA6/8/9/46/45/47) including sublobar/claustrum and anterior cingulate gyrus (BA24), their activation extending to the prefrontal area; (2) right parietal lobe (BA7/39/40); and (3) ventral occipitotemporal cortex including fusiform gyrus (BA37/20) and inferior occipital cortex (BA18/19).

As shown in Table 1 and Figure 3, when comparing recognition versus baseline $e_{2}$, the activation pattern in bilateral cortices included frontal cortex (BA6/8/9/10/13/45/46/47), posterior cingulate gyrus (BA31), parietal lobe (BA7/40), and cuneus (BA18/19). However, there were limited activities in left cortices. Significant activations included two clusters in different regions: (1) bilateral superior medial frontal and (2) occipitoparietal regions. Strong activation was located in the right superior frontal gyrus with a peak activation area in the 
TABLE 1: Stereotactic Talairach coordinates, $t$-values, volume, and corresponding BAs for significantly activated regions (encoding versus baseline $_{1}$ and recognition versus baseline ${ }_{2}$ ).

\begin{tabular}{|c|c|c|c|c|c|}
\hline Comparison & Activated regions & $\mathrm{BA}$ & Coordinates & Peak $t$-values & Volume $\left(\mathrm{mm}^{3}\right)$ \\
\hline \multirow{10}{*}{ Encoding versus baseline $_{1}$} & R. inferior frontal gyrus & $13 / 45 / 47$ & $36,24,7$ & 9.20 & 4,941 \\
\hline & R. middle frontal gyrus & $46 / 9 / 8$ & $56,28,26$ & 6.42 & 7,857 \\
\hline & R. superior frontal gyrus & 6 & $3,11,55$ & 7.59 & 5,211 \\
\hline & L. middle frontal gyrus & 10 & $-33,56,29$ & 6.27 & 2,403 \\
\hline & R. claustrum & - & $24,26,4$ & 6.49 & 4,536 \\
\hline & Cingulate gyrus & 24 & $3,4,25$ & 4.79 & 2,916 \\
\hline & R. inferior parietal lobule & $39 / 40$ & $33,-62,42$ & 6.28 & 4,401 \\
\hline & R. superior parietal lobule & 7 & $27,-53,44$ & 5.43 & 5,778 \\
\hline & R. fusiform gyrus & $37 / 20$ & $42,-62,-22$ & 4.99 & 7,668 \\
\hline & R. inferior occipital gyrus & $18 / 19$ & $30,-93,-3$ & 5.39 & 4,914 \\
\hline \multirow{8}{*}{ Recognition versus baseline $_{2}$} & R. superior frontal gyrus & 6 & $6,9,58$ & 8.54 & 7,020 \\
\hline & L. superior medial frontal gyrus & $8 / 6 / 9 / 10 / 46$ & $0,20,46$ & 6.69 & 5,373 \\
\hline & L. inferior frontal gyrus & $13 / 45 / 47$ & $-33,24,7$ & 6.68 & 3,078 \\
\hline & L. claustrum & - & $-27,21,13$ & 5.90 & 2,565 \\
\hline & R. superior parietal lobule & 7 & $33,-50,52$ & 4.72 & 3,645 \\
\hline & R. inferior parietal lobule & 40 & $39,-42,33$ & 4.30 & 2,592 \\
\hline & Cingulate gyrus & 31 & $-18,-63,14$ & 5.28 & 4,671 \\
\hline & R. cuneus & $18 / 19$ & $6,-81,12$ & 6.57 & 6,561 \\
\hline
\end{tabular}

L, left hemisphere; R, right hemisphere; BAs: Brodmann's areas.

Coordinates $(x, y, z)$ are shown in Talairach space for the peak activation of each activated region. Peak $t$-values: peak intensity of voxel ( $t$-score of peak voxel). Volume: numbers of voxel for the center-mass of peak activated region.

TABLE 2: Stereotactic Talairach coordinates, $t$-values, volume, and corresponding BAs for significantly activated regions (encoding versus recognition, recognition versus encoding).

\begin{tabular}{|c|c|c|c|c|c|}
\hline Comparison & Activated regions & $\mathrm{BA}$ & Coordinates & Peak $t$-values & Volume $\left(\mathrm{mm}^{3}\right)$ \\
\hline \multirow{6}{*}{ Encoding versus recognition } & L. inferior occipital gyrus & $18 / 19$ & $-30,-96,-3$ & 9.22 & 7,965 \\
\hline & R. midinferior occipital gyrus & $18 / 19$ & $27,-90,-1$ & 8.78 & 13,014 \\
\hline & L. fusiform gyrus & $37 / 20 / 36$ & $-39,-56,-12$ & 5.16 & 16,848 \\
\hline & R. fusiform gyrus & $37 / 20$ & $42,-53,-10$ & 6.78 & 21,762 \\
\hline & L. inferior frontal gyrus & 47 & $-15,31,-17$ & 5.23 & 1,890 \\
\hline & R. superior medial frontal gyrus & $11 / 25$ & $21,40,-20$ & 5.11 & 4,671 \\
\hline \multirow{8}{*}{ Recognition versus encoding } & L. precuneus & 7 & $-3,-73,45$ & 8.50 & 12,285 \\
\hline & R. precuneus & 7 & $6,-71,39$ & 8.06 & 15,417 \\
\hline & Cingulate gyrus & $31 / 32$ & $-18,-60,17$ & 7.57 & 12,285 \\
\hline & L. medial frontal gyrus & 10 & $-6,61,5$ & 7.26 & 10,827 \\
\hline & R. superior medial frontal gyrus & 10 & $30,56,22$ & 7.03 & 6,615 \\
\hline & L. middle temporal lobe & 21 & $-45,-4,-12$ & 4.95 & 8,559 \\
\hline & R. superior temporal gyrus & 22 & $59,12,-1$ & 6.40 & 3,078 \\
\hline & L. inferior occipital gyrus & $18 / 19$ & $-18,-95,27$ & 5.69 & 8,181 \\
\hline
\end{tabular}

L, left hemisphere; R, right hemisphere; BAs: Brodmann's areas.

Coordinates $(x, y, z)$ are shown in Talairach space for the peak activation of each activated region. Peak $t$-values: peak intensity of voxel ( $t$-score of peak voxel). Volume: numbers of voxel for the center-mass of peak activated region.

premotor cortex (BA6, coordinates: $6,9,58, t$-value $=8.54$, and $P<0.05$ ).

The detailed brain regions of significant activation for encoding versus recognition are presented in Table 2 and Figure 4. Compared with recognition, encoding activated large clusters in bilateral ventral occipitotemporal cortex (BA18/19/37/20/36) with a peak activation area in left inferior occipital gyrus (BA18) (coordinates: $-30,-96,-3, t$-value $=$
9.22, and $P<0.05)$. Significant activations included two clusters in different regions: (1) ventral occipitotemporal cortex and (2) inferior and medial frontal cortices (BA47/11/25).

As shown in Table 2 and Figure 5, compared with encoding, recognition significantly activated three clusters in different regions: (1) bilateral precuneus (BA7), (2) cingulate gyrus (BA31/32), and (3) superior medial frontal gyrus (BA10). Strong activation was located in the left superior parietal 


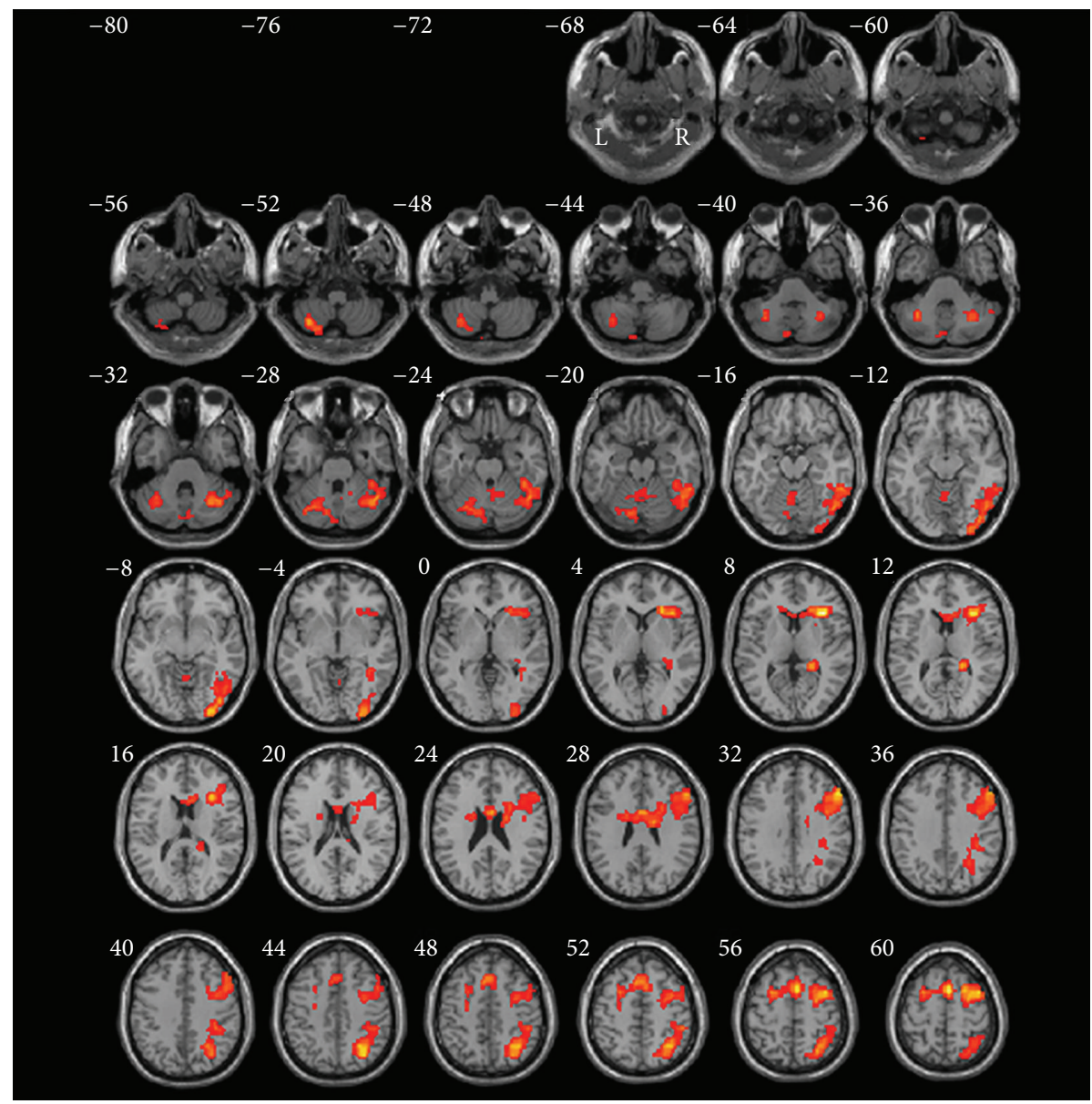

FIgURE 2: Normalized brain activation maps averaged over 14 participants at a threshold of $P<0.05$ of encoding versus baseline ${ }_{1}$. Talairach $Z$ coordinates are given above each horizontal section. Combination threshold of voxel was set at $P<0.05$, and a cluster size $>389$ corresponded to a corrected $P<0.05$. This correction was determined using the AlphaSim software in AFNI. L, left hemisphere; R, right hemisphere.

gyrus with a peak activation area in precuneus (BA7, coordinates: $-3,-73,45, t$-value $=8.50$, and $P<0.05)$.

\section{Discussion}

The present fMRI study aimed to explore neural substrates of integrative semantic categories in a more holistic manner. In contrast to previous studies, we used category-related logogram pairs to contribute to deep semantic strategies for encoding and recognition tasks. To ensure that brain activation was specifically attributed to semantic processing and not to categorical processing, semantics and control conditions were matched for orthographic or phonological (in Chinese) processing demands, and all four categories were pooled together. Logogram pairs from four different categories (fruits, animals, tools, and cloths) were included in a single experiment and analyzed as an entity to examine cerebral activity at a relatively integrative level rather than at a specific level.

The tasks employed in the present study activated ventral occipitotemporal, inferior frontal, superior frontal gyrus, and occipitoparietal regions, but not the posterior left inferior frontal gyrus (LIFG) (BA44/45), which is commonly activated in semantic memory tasks. The role of the LIFG remains unclear. Some studies claimed that the anterior LIFG (BA47) plays an important role in semantic processing [18], whereas the posterior LIFG (BA44/45) is specialized for phonological processing [19]. The data presented above suggest that the anterior region is associated with semantic processing, irrespective of phonological demands. The LIFG has been previously shown to be involved in generating semantic associations [20, 21], particularly while making decisions concerning semantic associations [22-24]. Another explanation of this modulatory response is that it reflects increased demand for selection between categorical associations [25-27]. In the semantic tasks used in the present study, the subjects were required to select the related features of the logogram pairs to generate categorical associations. Therefore, our findings support the idea that the LIFG is involved in selecting among competing semantic features stored in the cortex [28].

The dorsolateral prefrontal cortex (DLPFC) is roughly equivalent to BA9 and BA46 and plays an important role in processing mnemonic information, for example, working memory. Some fMRI data have shown that the DLPFC activity is higher during semantic relationship-encoding 


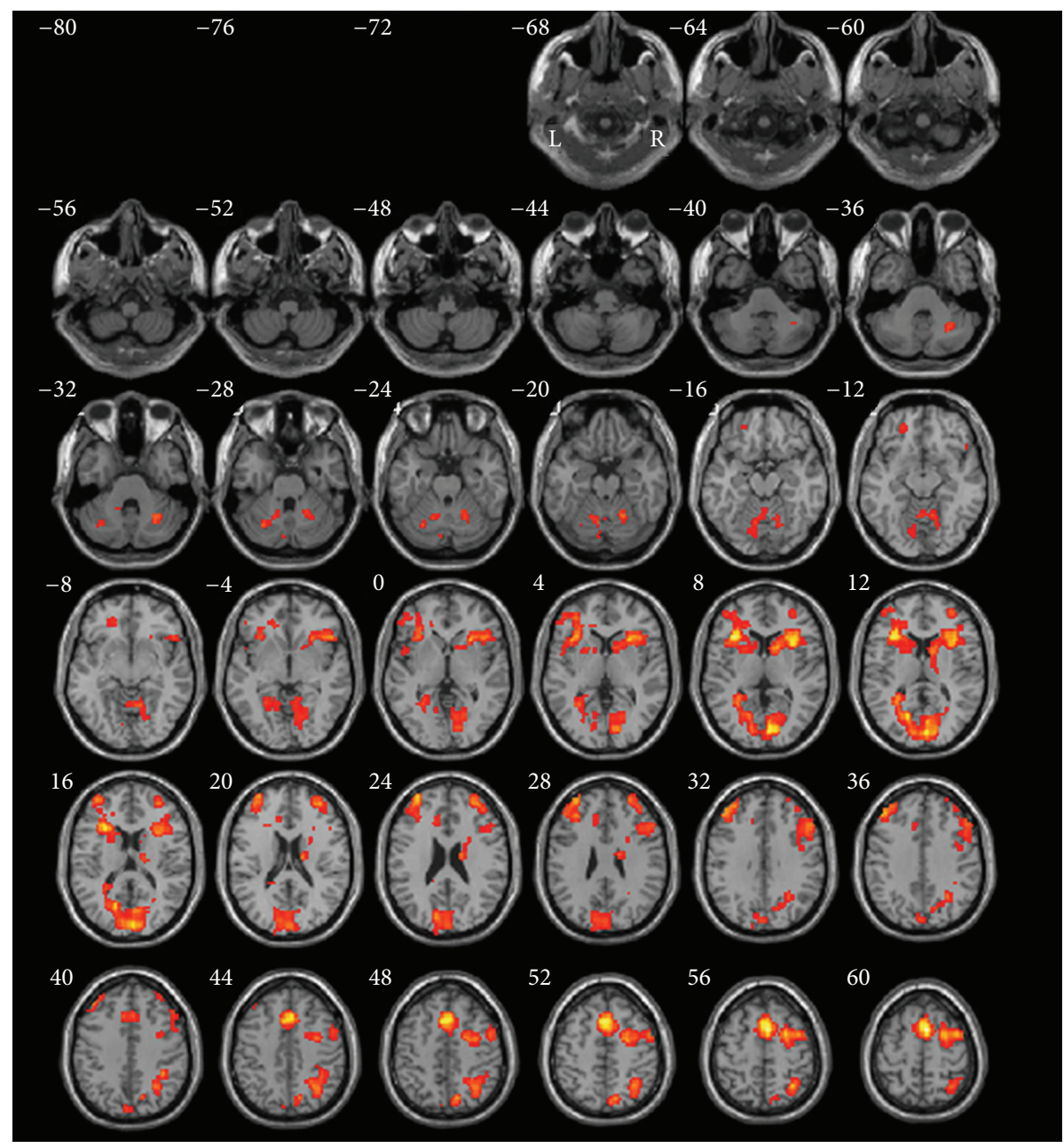

Figure 3: Normalized brain activation maps averaged over 14 participants at a threshold of $P<0.05$ of recognition versus baseline ${ }_{2}$. Talairach $Z$ coordinates are given above each horizontal section. Combination threshold of voxel was set at $P<0.05$, and a cluster size $>389$ corresponded to a corrected $P<0.05$. This correction was determined using the AlphaSim software in AFNI. L, left hemisphere; R, right hemisphere.

processing compared with item-specific encoding [29-31], indicating that the DLPFC preferentially modulates semantic relational processing. In this study, subjects were required to build semantic relations between items using categorycorrelated features. Thus, it may be inferred that left BA9/46 activation may contribute to modulating semantic relational processing, as might be suggested by previous studies [32, 33]. Nevertheless, these results are similar to a previous study showing that BA9/46 is involved in the recognition of Japanese logograms [34].

As shown above, the total extent of brain activation, particularly in lateral-ventral regions of the occipitotemporal cortex, was significantly larger for the encoding process compared with the recognition one. These results indicate that the neurocognitive mechanisms of semantic processing underlying encoding may differ from those behind recognition [35]. Goodale and Milner [36] proposed a division of labor in the visual pathways of primate cerebral cortex between a ventral stream that contributes to perception of the visual world and a dorsal stream specialized for the visual control of action. In the current study, lateral and ventral occipitotemporal regions displayed increased levels of encoding-related activity when the subjects were required to encode logogram pairs. However, we found little evidence for ATL involvement in semantic processing; activation was only observed in the ventral-lateral regions of posterior temporal cortex. This is consistent with a report showing that normal individuals display strong activation of ventral-lateral regions of posterior temporal cortex during a categorical association task, whereas patients with ATL atrophy failed to show activity in this region [37].

Our data suggest that the encoding task relies on featuregeneral processes to identify target word pairs for memorization and feature-specific processes to encode the pairing, while the retrieval task only relies on feature-specific processes for successfully retrieving the response words. The direct contrast between these two tasks might reflect the feature-general processes of semantic representation. 


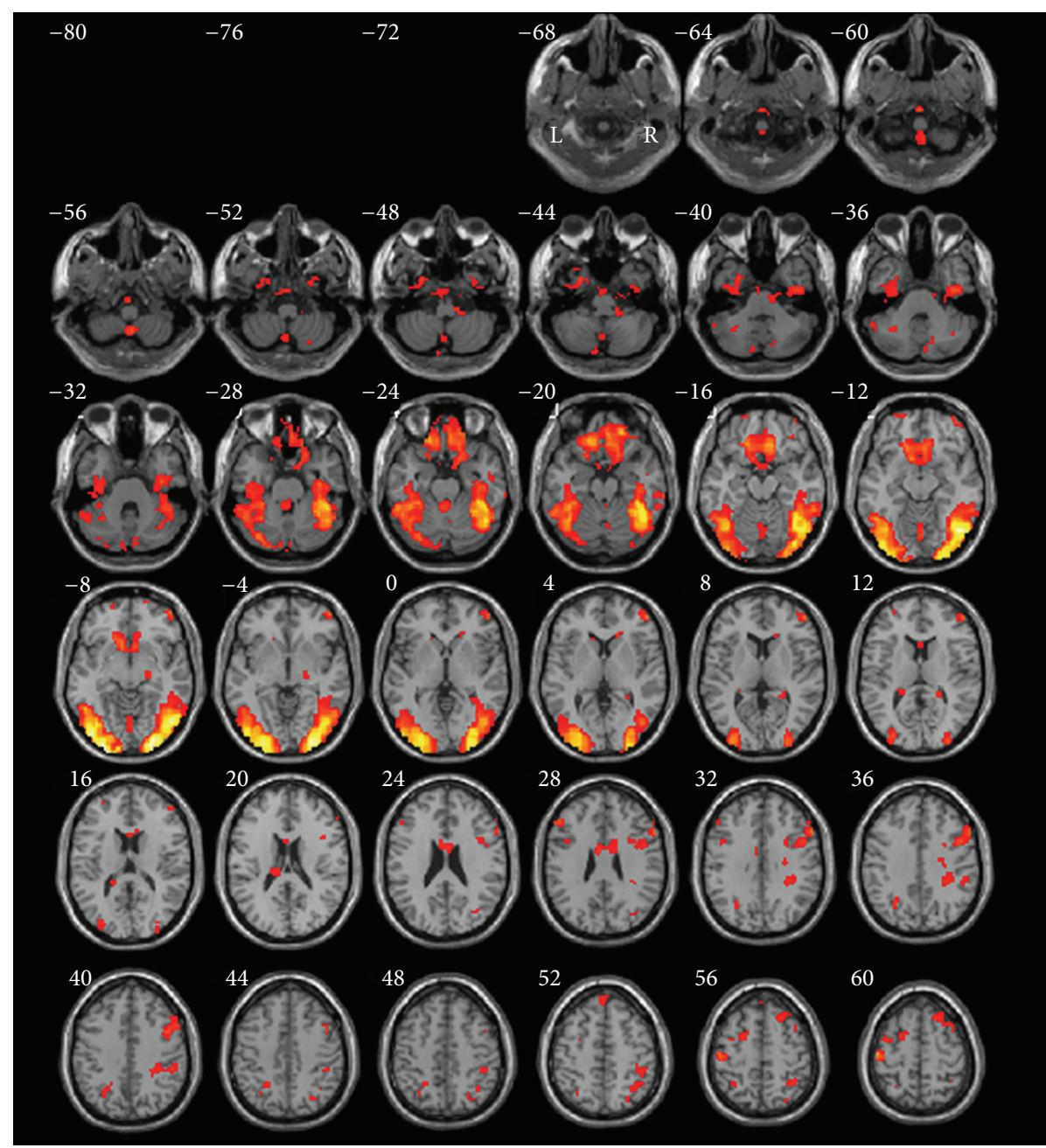

FIgURE 4: Normalized brain activation maps averaged over 14 participants at a threshold of $P<0.05$ of encoding versus recognition. Talairach $Z$ coordinates are given above each horizontal section. Combination threshold of voxel was set at $P<0.05$, and a cluster size $>389$ corresponded to a corrected $P<0.05$. This correction was determined using the AlphaSim software in AFNI. L, left hemisphere; R, right hemisphere.

However, further study using Chinese characters is necessary to complement these results. A previous report proposed that adults more effectively engage right hemisphere brain regions involved in the visual-spatial analysis of Chinese characters [38], indicating that Chinese character processing is age related. In addition, semantic and visual errors were shown to be associated with young age and low education level in children [39]. Therefore, in this study, we selected subjects with similar ages and education levels, with the examination process clearly explained to all subjects, to minimize the effects of these confounding factors. Further studies will focus on populations of various ages as well as different language comprehension and education levels.

The present study suffers from some limitations. It was not exhaustive in semantic scope since all four categories were pooled to only keep the effect of encoding and recognizing logograms. It would be interesting to test subjects with the same method on different tasks, such as categorically related picture pairs. It would also be useful to examine the anatomic and functional connectivity of the ROIs to identify the directional and effective connections between ROIs, thus determining how ROIs interact with each other within a broader network. Such connectivity analysis can be explored to assess whether anatomic connectivity affects functional connectivity. Finally, it would be interesting to use the same alphabetical materials in English to test native English speakers and further investigate whether language surface features affect semantic processing representations. In addition, an increase in sample size would further validate and support the findings of this study.

\section{Conclusions}

Overall, our results suggested that distributed networks of discrete cortical regions with distinct roles are active during semantic processing of logograms. The ventral occipitotemporal and inferior frontal regions display increased levels of encoding-related activity. The dorsal medial brain regions 


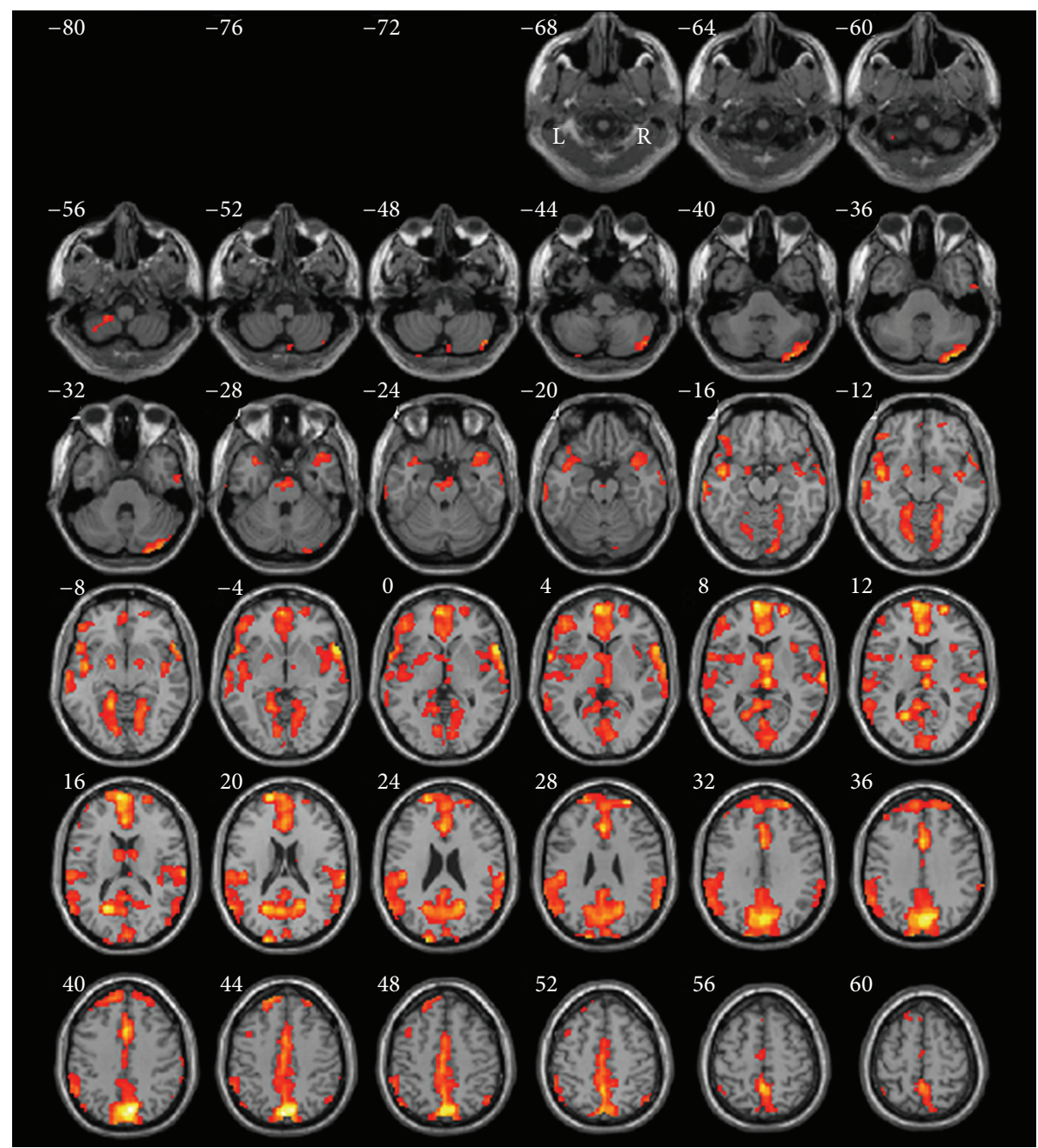

Figure 5: Normalized brain activation maps averaged over 14 participants at a threshold of $P<0.05$ of recognition versus encoding. Talairach $Z$ coordinates are given above each horizontal section. Combination threshold of voxel was set at $P<0.05$, and a cluster size $>389$ corresponded to a corrected $P<0.05$. This correction was determined using the AlphaSim software in AFNI. L, left hemisphere; R, right hemisphere.

including superior frontal gyrus and occipitoparietal regions are associated with recognition-related activity.

\section{Conflict of Interests}

The authors declare that there is no conflict of interests regarding the publication of this paper.

\section{Acknowledgment}

This study was funded by the National Natural Science Foundation of China (Grant nos. 81271524 and 81371514).

\section{References}

[1] K. Patterson, P. J. Nestor, and T. T. Rogers, "Where do you know what you know? The representation of semantic knowledge in the human brain," Nature Reviews Neuroscience, vol. 8, no. 12, pp. 976-987, 2007.
[2] L. H. Tan, A. R. Laird, K. Li, and P. T. Fox, "Neuroanatomical correlates of phonological processing of Chinese characters and alphabetic words: a meta-analysis," Human Brain Mapping, vol. 25, no. 1, pp. 83-91, 2005.

[3] L. H. Tan, J. A. Spinks, C.-M. Feng et al., "Neural systems of second language reading are shaped by native language," Human Brain Mapping, vol. 18, no. 3, pp. 158-166, 2003.

[4] A. Ishai, L. G. Ungerleider, A. Martin, and J. V. Haxby, "The representation of objects in the human occipital and temporal cortex," Journal of Cognitive Neuroscience, vol. 12, supplement 2, pp. 35-51, 2000.

[5] M. L. Kellenbach, M. Brett, and K. Patterson, "Large, colorful, or noisy? Attribute- and modality-specific activations during retrieval of perceptual attribute knowledge," Cognitive, Affective \& Behavioral Neuroscience, vol. 1, no. 3, pp. 207-221, 2001.

[6] A. Martin, J. V. Haxby, F. M. Lalonde, C. L. Wiggs, and L. G. Ungerleider, "Discrete cortical regions associated with knowledge of color and knowledge of action," Science, vol. 270, no. 5233, pp. 102-105, 1995. 
[7] A. Martin, C. L. Wiggs, L. G. Ungerleider, and J. V. Haxby, "Neural correlates of category-specific knowledge," Nature, vol. 379, no. 6566, pp. 649-652, 1996.

[8] P. Vitali, J. Abutalebi, M. Tettamanti et al., "Generating animal and tool names: an fMRI study of effective connectivity," Brain and Language, vol. 93, no. 1, pp. 32-45, 2005.

[9] J. T. Devlin, R. P. Russell, M. H. Davis et al., "Is there an anatomical basis for category-specificity? Semantic memory studies in PET and fMRI," Neuropsychologia, vol. 40, no. 1, pp. 54-75, 2002.

[10] L. Mo, H.-L. Liu, H. Jin, and Y.-L. Yang, "Brain activation during semantic judgment of Chinese sentences: a functional MRI study," Human Brain Mapping, vol. 24, no. 4, pp. 305-312, 2005.

[11] C. Gerlach, "A review of functional imaging studies on category specificity," Journal of Cognitive Neuroscience, vol. 19, no. 2, pp. 296-314, 2007.

[12] A. Martin and L. L. Chao, "Semantic memory and the brain: structure and processes," Current Opinion in Neurobiology, vol. 11, no. 2, pp. 194-201, 2001.

[13] P. J. Snyder and L. J. Harris, "Handedness, sex, and familial sinistrality effects on spatial tasks," Cortex, vol. 29, no. 1, pp. 115134, 1993.

[14] S. X. Lv and S. S. Deng, Xiandai Hanyu Cidian, The Commerical Press, Beijing, China, 2005.

[15] Y. Liu, Xiandai Hanyu Changyongci Cipin Cidian, China Astronautic Publishing House, Beijing, China, 1990.

[16] C.-G. Yan and Y.-F. Zang, "DPARSF: a MATLAB toolbox for 'pipeline' data analysis of resting-state fMRI," Frontiers in Systems Neuroscience, vol. 4, article 13, 2010.

[17] C. F. Beckmann, M. Jenkinson, and S. M. Smith, "General multilevel linear modeling for group analysis in FMRI," NeuroImage, vol. 20, no. 2, pp. 1052-1063, 2003.

[18] Y. Uchiyama, H. Toyoda, M. Honda et al., "Functional segregation of the inferior frontal gyrus for syntactic processes: a functional magnetic-resonance imaging study," Neuroscience Research, vol. 61, no. 3, pp. 309-318, 2008.

[19] S. Bookheimer, "Functional MRI of language: new approaches to understanding the cortical organization of semantic processing," Annual Review of Neuroscience, vol. 25, pp. 151-188, 2002.

[20] P. C. Fletcher, T. Shallice, and R. J. Dolan, "'Sculpting the response space'-an account of left prefrontal activation at encoding," NeuroImage, vol. 12, no. 4, pp. 404-417, 2000.

[21] R. A. Poldrack, A. D. Wagner, M. W. Prull, J. E. Desmond, G. H. Glover, and J. D. E. Gabrieli, "Functional specialization for semantic and phonological processing in the left inferior prefrontal cortex," NeuroImage, vol. 10, no. 1, pp. 15-35, 1999.

[22] L. J. Otten, R. N. A. Henson, and M. D. Rugg, "Depth of processing effects on neural correlates of memory encoding: relationship between findings from across- and within-task comparisons," Brain, vol. 124, no. 2, pp. 399-412, 2001.

[23] G. T. Stebbins, M. C. Carrillo, J. Dorfman et al., "Aging effects on memory encoding in the frontal lobes," Psychology and Aging, vol. 17, no. 1, pp. 44-55, 2002.

[24] A. D. Wagner, D. L. Schacter, M. Rotte et al., "Building memories: remembering and forgetting of verbal experiences as predicted by brain activity," Science, vol. 281, no. 5380, pp. 1188-1191, 1998.

[25] E. A. Hirshorn and S. L. Thompson-Schill, "Role of the left inferior frontal gyrus in covert word retrieval: neural correlates of switching during verbal fluency," Neuropsychologia, vol. 44, no. 12, pp. 2547-2557, 2006.
[26] I. P. Kan and S. L. Thompson-Schill, "Effect of name agreement on prefrontal activity during overt and covert picture naming," Cognitive, Affective \& Behavioral Neuroscience, vol. 4, no. 1, pp. 43-57, 2004.

[27] S. L. Thompson-Schill, "Neuroimaging studies of semantic memory: inferring 'how' from 'where," Neuropsychologia, vol. 41, no. 3, pp. 280-292, 2003.

[28] S. L. Thompson-Schill, M. D'Esposito, and I. P. Kan, "Effects of repetition and competition on activity in left prefrontal cortex during word generation," Neuron, vol. 23, no. 3, pp. 513-522, 1999.

[29] C. Hawco, J. L. Armony, and M. Lepage, "Neural activity related to self-initiating elaborative semantic encoding in associative memory," NeuroImage, vol. 67, pp. 273-282, 2013.

[30] N. M. Long, I. Öztekin, and D. Badre, "Separable prefrontal cortex contributions to free recall," The Journal of Neuroscience, vol. 30, no. 33, pp. 10967-10976, 2010.

[31] L. J. Murray and C. Ranganath, "The dorsolateral prefrontal cortex contributes to successful relational memory encoding," The Journal of Neuroscience, vol. 27, no. 20, pp. 5515-5522, 2007.

[32] A. M. Owen, K. M. McMillan, A. R. Laird, and E. Bullmore, "Nback working memory paradigm: a meta-analysis of normative functional neuroimaging studies," Human Brain Mapping, vol. 25, no. 1, pp. 46-59, 2005.

[33] K. B. McDermott, S. E. Petersen, J. M. Watson, and J. G. Ojemann, "A procedure for identifying regions preferentially activated by attention to semantic and phonological relations using functional magnetic resonance imaging," Neuropsychologia, vol. 41, no. 3, pp. 293-303, 2003.

[34] X. Li, J. Wu, and Q. Guo, "Differential activity of semantic and font size judgment processing of Japanese word: an fMRI study," in Proceedings of the 6th International Conference on Complex Medical Engineering (CME '12), pp. 677-682, IEEE, Kobe, Japan, July 2012.

[35] T. J. Spencer, D. Montaldi, Q.-Y. Gong, N. Roberts, and A. R. Mayes, "Object priming and recognition memory: dissociable effects in left frontal cortex at encoding," Neuropsychologia, vol. 47, no. 13, pp. 2942-2947, 2009.

[36] M. A. Goodale and A. D. Milner, "Separate visual pathways for perception and action," Trends in Neurosciences, vol. 15, no. 1, pp. 20-25, 1992.

[37] C. J. Mummery, K. Patterson, R. J. S. Wise, R. Vandenbergh, C. J. Price, and J. R. Hodges, "Disrupted temporal lobe connections in semantic dementia," Brain, vol. 122, no. 1, pp. 61-73, 1999.

[38] F. Cao, D. Peng, L. Liu et al., "Developmental differences of neurocognitive networks for phonological and semantic processing in Chinese word reading," Human Brain Mapping, vol. 30, no. 3, pp. 797-809, 2009.

[39] C. K. K. Chan and L. S. Siegel, "Phonological processing in reading Chinese among normally achieving and poor readers," Journal of Experimental Child Psychology, vol. 80, no. 1, pp. 2343, 2001. 


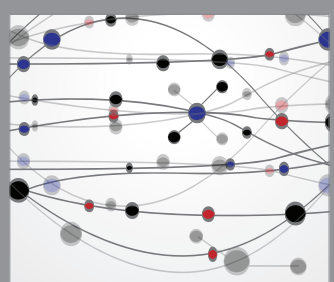

The Scientific World Journal
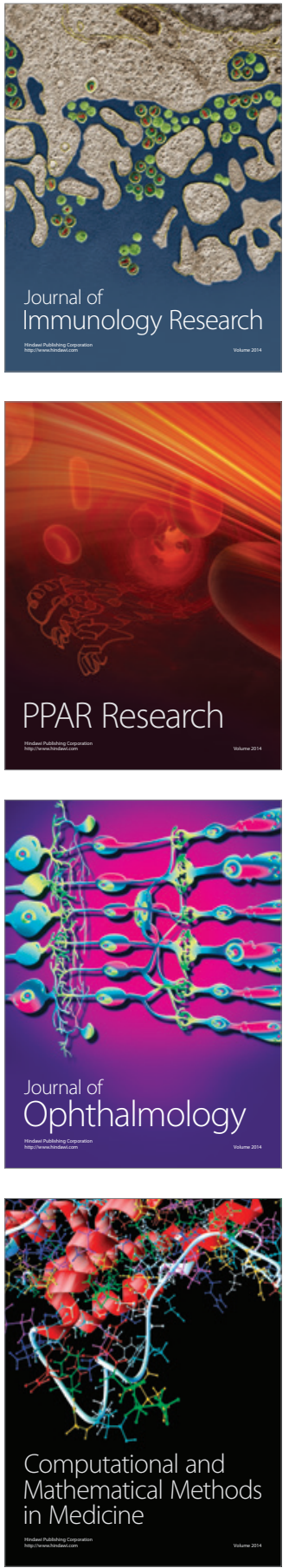

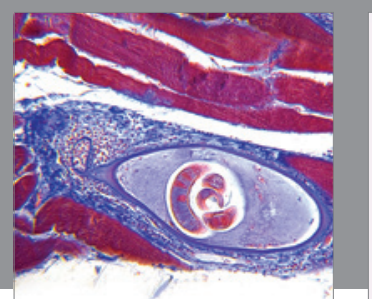

Gastroenterology Research and Practice

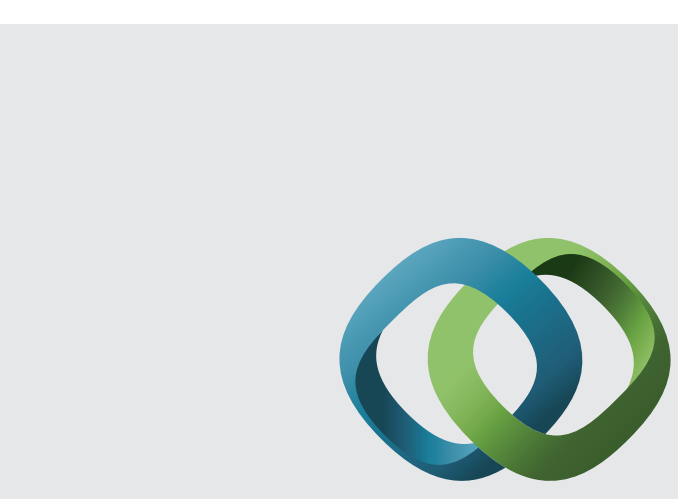

\section{Hindawi}

Submit your manuscripts at

http://www.hindawi.com
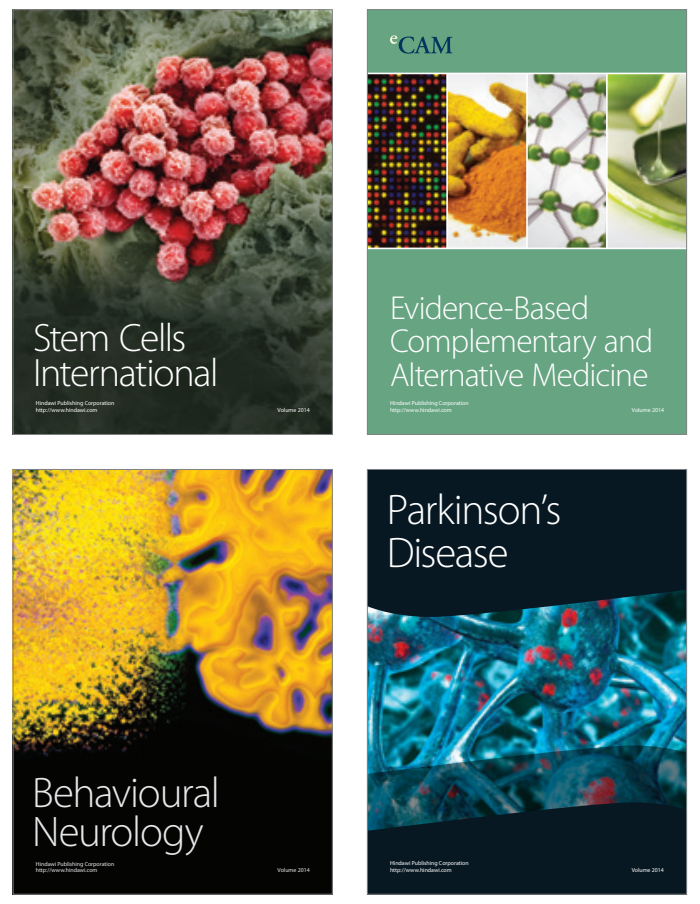
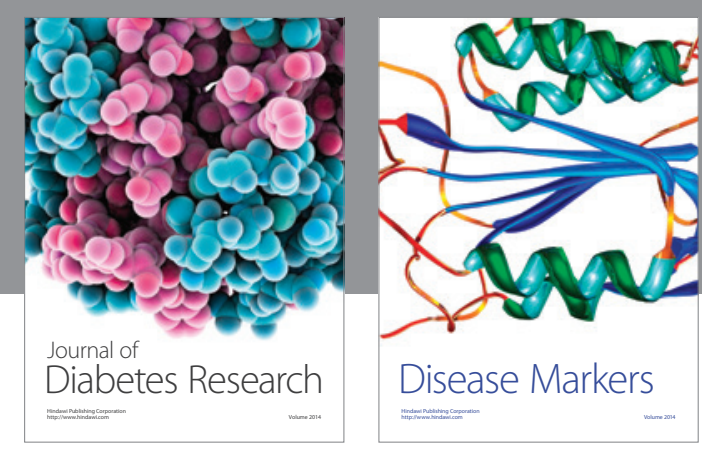

Disease Markers
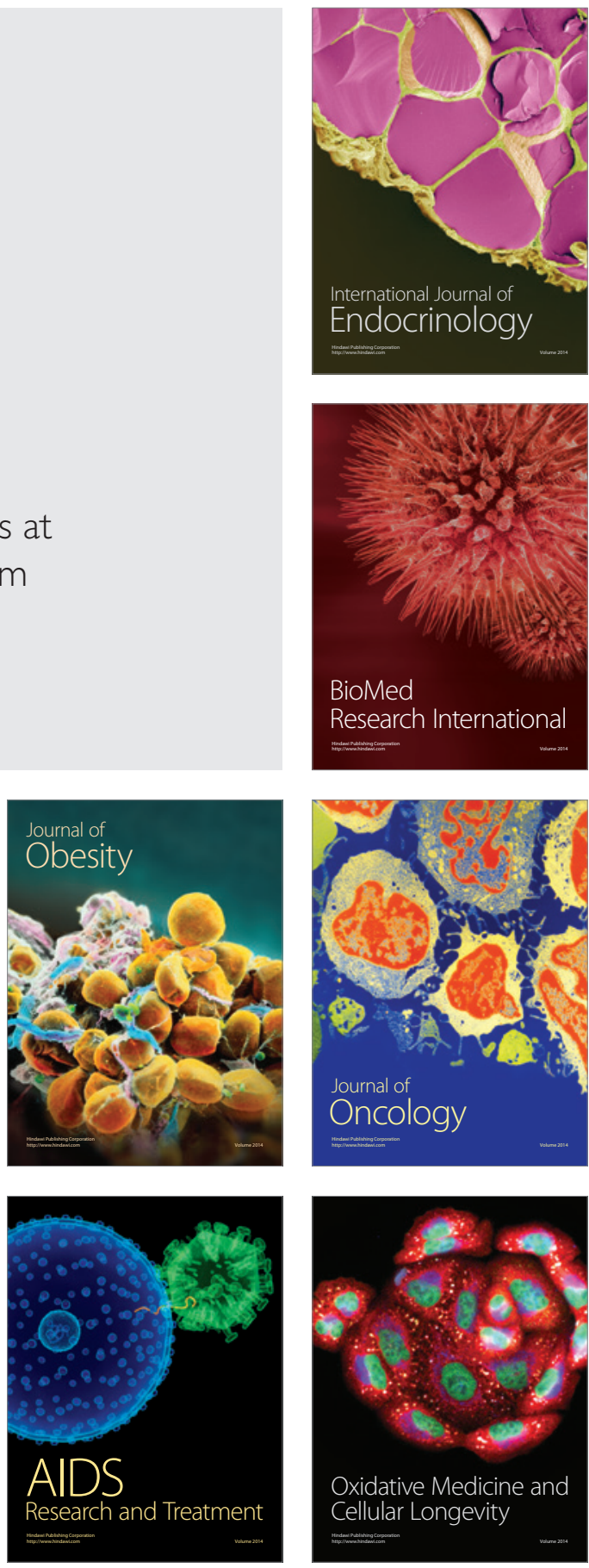